\title{
Comment: Data quality
}

One of the key issues to affect - and, in many cases, to hinder - the development of good customer management is data quality. Senior managers will spend months choosing between systems of near identical functionality. They will spend millions on installing the latest contact management technology (or CRM, or customer experience). Then, when data, data quality and the associated processes arrive on the agenda, they delegate to their subordinates - or just assume that 'it'll be alright on the night'. At which point, all the millions invested might as well have been poured down the drain, because what they will end up with is 'business as usual' - albeit in a brand new, shinier wrapping.

The following pieces are insights from two companies that work in the area of identifying and limiting the damage caused by poor data. These are practice articles - which means that they will be extolling the virtues of their own solutions. The reader must therefore be aware of that and be aware too that the journal does not in any way endorse the products mentioned. By all means, explore for yourselves and, if you feel the product does not live up to the promise, we would be interested in hearing back from you.

Nonetheless, there is much truth in both of the pieces and lessons to be drawn from them.

A further irony of publishing quarterly - and of being but one of a range of journals - is that papers that strongly support a theme explored in one issue may end up in another journal. With that in mind, I would also recommend that readers who have a serious interest in this subject should obtain copies of a paper by Atai Winkler on the subject of inputting missing values in a data set. The paper is due to be published in Journal of Targeting, Measurement and Analysis for Marketing, Volume 13, Issue 2 and is entitled 'Maximising the value of your data'. That, too, begins with academic research and has resulted in the development of a product for filling gaps in data. Again, we are happy to vouch for the theory, whilst leaving it to you, the reader, to evaluate the product. 